\title{
Novel diagnostic tools for identifying cognitive impairment using olfactory- stimulated functional near-infrared spectroscopy: patient-level, single-group, diagnostic trial
}

Jaewon Kim ${ }^{1+}$, Dong Keon Yon ${ }^{2,3+} \mathbb{D}$, Kyu Yeong Choi ${ }^{4,5}$, Jang Jae Lee ${ }^{4}$, Namwoo Kim$^{6}$, Kun Ho Lee ${ }^{4,7,8^{*}+}$ and Jae Gwan Kim ${ }^{1 *+}$

\begin{abstract}
Introduction: Basic studies suggest that olfactory dysfunction and functional near-infrared spectroscopy (fNIRS) can be used as tools for the diagnosis of mild cognitive impairment (MCl); however, real-world evidence is lacking. We investigated the potential diagnostic efficacy of olfactory-stimulated fNIRS for early detection of $\mathrm{MCl}$ and/or Alzheimer disease (AD).

Methods: We conducted a patient-level, single-group, diagnostic interventional trial involving elderly volunteers (age $>60$ years) suspected of declining cognitive function. Patients received open-label olfactory-stimulated fNIRS for measurement of oxygenation difference in the orbitofrontal cortex. All participants underwent amyloid PET, MRI, Mini-Mental State Examination (MMSE), and Seoul Neuropsychological Screening Battery (SNSB).

Results: Of 97 subjects, 28 (28.9\%) were cognitively normal, 32 (33.0\%) had preclinical AD, 21 (21.6\%) had MCl, and 16 (16.5\%) had AD. Olfactory-stimulated oxygenation differences in the orbitofrontal cortex were associated with cognitive impairment; the association was more pronounced with cognitive severity. Olfactory-stimulated oxygenation difference was associated with MMSE (adjusted $\beta[a \beta] 1.001 ; 95 \% \mathrm{Cl} 0.540-1.463$ ), SNSB language and related function $(a \beta, 1.218 ; 95 \% C l, 0.020-2.417)$, SNSB memory $(a \beta, 1.963 ; 95 \% C l, 0.841-3.084)$, SNSB frontal/executive function $(a \beta, 1.715 ; 95 \% \mathrm{Cl}, 0.401-3.029)$ scores, standard uptake value ratio from amyloid PET ( $a \beta,-10.083 ; 95 \% \mathrm{Cl},-19.063$ to -1.103$)$, and hippocampal volume from MRI $(a \beta, 0.002 ; 95 \% C l, 0.001-0.004)$. Olfactory-stimulated oxygenation
\end{abstract}

*Correspondence: leekho@chosun.ac.kr; jaekim@gist.ac.kr

${ }^{\dagger} J a e w o n$ Kim and Dong Keon Yon contributed equally as first authors.

${ }^{\dagger}$ Dong Keon Yon contributed as senior author.

${ }^{\dagger}$ Kun Ho Lee and Jae Gwan Kim contributed equally as corresponding authors.

${ }^{1}$ Department of Biomedical Science and Engineering, Gwangju Institute

of Science and Technology, Gwangju, Republic of Korea

${ }^{7}$ Gwangju Alzheimer's \& Related Dementia Cohort Research Center,

Department of Biomedical Science, Chosun University, Gwangju 61452,

Republic of Korea

Full list of author information is available at the end of the article

(c) The Author(s) 2022. Open Access This article is licensed under a Creative Commons Attribution 4.0 International License, which permits use, sharing, adaptation, distribution and reproduction in any medium or format, as long as you give appropriate credit to the original author(s) and the source, provide a link to the Creative Commons licence, and indicate if changes were made. The images or other third party material in this article are included in the article's Creative Commons licence, unless indicated otherwise in a credit line to the material. If material is not included in the article's Creative Commons licence and your intended use is not permitted by statutory regulation or exceeds the permitted use, you will need to obtain permission directly from the copyright holder. To view a copy of this licence, visit http://creativecommons.org/licenses/by/4.0/. The Creative Commons Public Domain Dedication waiver (http://creativeco mmons.org/publicdomain/zero/1.0/) applies to the data made available in this article, unless otherwise stated in a credit line to the data. 
difference in the orbitofrontal cortex was superior in diagnosing $\mathrm{MCl}$ and $\mathrm{AD}$ ( $\mathrm{AUC}, 0.909 ; 95 \% \mathrm{Cl}, 0.848-0.971$ ), compared to amyloid PET (AUC, $0.793 ; 95 \%$ Cl, 0.694-0.893) or MRI (AUC, $0.758 ; 95 \%$ Cl, $0.644-0.871$ ).

Discussion: Our trial showed that olfactory-stimulated oxygenation differences in the orbitofrontal cortex detected by fNIRS were associated with cognitive impairment and cognitive-related objectives. This novel approach may be a potential diagnostic tool for patients with $\mathrm{MCl}$ and/or AD.

Trial registration: CRIS number, KCT0006197.

Keywords: Cognitive impairment, Alzheimer's disease, fNIRS, Mild cognitive impairment

\section{Background}

Alzheimer's disease (AD) is the most common type of dementia worldwide $[1,2]$. Because of the absence of a definitive treatment method, the current alternative is to diagnose early, detect degenerative changes before they become severe, and delay cognitive decline as much as possible. A decrease in olfactory function before the onset of dementia has been shown in previous studies [3-5]. The olfactory function that is degraded in dementia is not the function of smell, but that of distinguishing odors, and the cause of this degradation is the formation of plaques and entanglements in the olfactory bulb and the inner olfactory cortex, which is involved in memory formation [3-5]. In animal and human autopsy studies, these plaques and tangles have been shown to occur earlier in the posterior nerves than in the cerebral cortex [6]. Additionally, a previous animal study conducted on $\operatorname{Tg} 2576$ mice demonstrated a decrease in dopaminergic neurons in the olfactory bulb resulting in overproduction of beta-amyloid precursors, which may lead to a decrease in olfactory discrimination function; the accumulation of beta-amyloid in the olfactory bulb was confirmed upon autopsy of the mice $[7,8]$. Therefore, if the olfactory function can be quantitatively measured, it can be detected at the stage of mild cognitive impairment (MCI).

Functional near-infrared spectroscopy (fNIRS) has the unique property of passing light through organ tissues and is subsequently absorbed by hemoglobin in the cerebral cortex, which enables real-time monitoring of hemodynamic changes in the cerebral cortex [9]. In particular, the difference in absorbance spectra between oxy-hemoglobin and deoxy-hemoglobin in the cortical regions can reflect continuous hemodynamic changes, which is useful as a marker of cerebral activity [9]. fNIRS has been used to perform functional activation in the field of neuropsychiatric disorders such as schizophrenia, developmental disorders, affective disorders, and dementia $[9,10]$.

In this context, fNIRS has multiple benefits over functional magnetic resonance imaging (fMRI), positron emission tomography (PET), and questionnaires for early detection of MCI and/or AD [11]. Previous researchers have suggested that the fNIRS approach stimulated by dual-task walking, N-back task, verbal fluency task, and memory and visuospatial test can be useful for the diagnosis of MCI and dementia [12-17] but these methods can increase the burden of medical providers and patients. However, the olfactory-stimulated fNIRS approach is a novel diagnostic method in which seven photodiodes are attached to the forehead of the patient during the experiment for only $1 \mathrm{~min}$ for each cycle (total $3 \mathrm{~min}$ for three cycles). The convenience of medical providers, short examination times, and non-invasive nature of the test can be useful to patients and medical providers. This novel diagnostic method is non-invasive, highly portable, has low cost and radiation, requires short examination time, and has fewer constraints on elderly patients who have difficulty sitting down for a long time or filling out questionnaires for examination. These benefits of novel fNIRS techniques provide a potential non-invasive and non-expensive alternative diagnostic methodology or therapeutic monitoring to fMRI, PET, and questionnaires for real-world clinical settings. However, the diagnostic study of fNIRS was limited by the relatively small study population, low-evidence study design, and non-standardization, such as stimulation types and interpretation methods.

Here, we hypothesized that olfactory-stimulated fNIRS is a novel diagnostic tool for the early detection of MCI and/or AD compared with conventional imaging studies. Through a patient-level, single-group, diagnostic intervention trial, we investigated the potential diagnostic efficacy of olfactory-stimulated fNIRS for early detection of $\mathrm{MCI}$ and/or $\mathrm{AD}$ and provided a standardized diagnostic protocol for olfactory-stimulated fNIRS. Furthermore, we aimed to clarify the diagnostic superiority of fNIRS in patients with $\mathrm{MCI}$ and/or AD in a real-world clinical setting.

\section{Material and methods Study design}

This study was designed as a prospective, patient-level, single-group, diagnostic accuracy study conducted in elderly volunteers (age $>60$ years) suspected of declining cognitive function between March 02 and August 30,2021 . Elderly volunteers (age $>60$ years) suspected of declining cognitive function were defined as those who 
had been recommended a test for cognitive function by physicians or related medical practitioners but had not yet undergone the test. The trial recruited those from a local community in Gwangju Metropolitan City, South Korea. Candidates were excluded if they had any severe life-threatening disease such as any malignancy or severe head trauma, a physical nasal obstruction with an inability to smell, or an alcohol or drug abuse problem, mental illness with psychosis, severe traumatic brain injury, major depressive disorder, or any other medical or psychological condition that, in the opinion of the investigator, may interfere with assessment of the main result or lead to non-cooperation while answering the questionnaire. Written informed consent was obtained from each participant and legal guardian at the time of enrollment. The study protocol was approved by the Institutional Review Board of the Gwangju Institute of Science and Technology (20210115-HR-58-01-02). The trial was registered with the Clinical Research Information Service of the Republic of Korea (CRIS number: KCT0006197). Our study adhered to the tenets of the Declaration of Helsinki.

\section{Participants}

We recruited 97 elderly volunteers (age $>60$ years) with a suspected decline in cognitive function. We assessed all participants by medical interviews with a detailed questionnaire to obtain baseline data on age, gender, education status, occupation, household income, smoking status, and the Charlson comorbidity index score. Cognitive function tests were performed using the MiniMental State Examination (MMSE), the Seoul Neuropsychological Screening Battery (SNSB) [18], and the Korean Instrumental Activities of Daily Living (K-IADL) [19], which are commonly used in cognitive function tests in South Korea. Cognitive impairment was defined as a $z$-score (normalized for age and education level) less than -1.0 on at least two of the SNSB tests, which assessed attention, language and related function, visuospatial function, memory, and frontal/executive function (Jak/ Bondi comprehensive criteria) $[18,20]$. Impairments of daily functioning were defined as a K-IADL score of less than 0.40 [19]. Body mass index was measured, and the apolipoprotein $\mathrm{E}(A P O E)$ genotype was tested from peripheral blood samples, with genotyping performed by extracting two SNP genomes from each individual. All participants underwent three-dimensional brain imaging (MPRAGE; TR, $2300 \mathrm{~ms}$; TE, $2.143 \mathrm{~ms}$; TI, $900 \mathrm{~ms}$; $F A, 9^{\circ}$; Fo V , $256 \times 256$; matrix, $320 \times 320$; slice thickness, $0.8 \mathrm{~mm}$ ) using a $3.0 \mathrm{~T}$ magnetic resonance (MR) scanner (MAGNETOM Skyra, Siemens Healthineers, Germany). The hippocampal volume was measured from each brain's MR image using the standard recon-all processing pipeline (FreeSurfer Version 5.3.0, Martinos Center for Biomedical Imaging, USA). In addition, ${ }^{18} \mathrm{~F}$-Florbetaben PET amyloid imaging (Discovery STE PET-CT scanner, GE Medical Systems, USA) was performed for all participants. The standard uptake value ratio (SUVR) was calculated and normalized by the cortical amyloid burden from six predefined cortical regions, including the anterior and posterior cingulate, frontal and lateral parietal, and lateral temporal regions with reference to the whole cerebellum [21, 22]. We used an Alzheimer's Disease Neuroimaging Initiative guideline SUVR cutoff of 1.1 to consider amyloid positivity [23].

The diagnostic criteria for MCI and Alzheimer's dementia for each group were based on the 2011 National Institute on Aging-Alzheimer's Association recommendations [24]. Although the criteria for patients with mild cognitive impairment met the core clinical criteria of the NIA-AA, patients were not tested for tau protein. Therefore, the subjects were classified as mild cognitive impairment with moderate probability based on Alzheimer's mild cognitive impairment criteria for research. Dementia patients were classified according to the NIA-AA diagnostic guideline of probable Alzheimer's dementia. Participants were classified into four groups: 55 (56.7\%) cognitively normal (CN) participants, $26(26.8 \%) \mathrm{MCI}$ patients, and 16 (16.5\%) AD patients.

\section{Diagnostic procedure}

To measure cortical activation, an fNIRS device was used while detecting olfactory stimulation (N.CER Co., Gwangju, South Korea). A 7-channel NIRS system (twowavelength LED [Fedy Tech, Shenzhen Fedy Technology Co., Ltd., China] and seven photodiodes [SFH 2201, OSRAM, Germany]) were used for all measurements; the light emitted can penetrate biological tissue and be absorbed by hemoglobin, which has different wavelengths $(695 \mathrm{~nm} \pm 20 \mathrm{~nm}$ and $830 \mathrm{~nm} \pm 20 \mathrm{~nm})$ when oxygenated and deoxygenated. Therefore, fNIRS can be used to identify cortical changes in oxygenated and deoxygenated hemoglobin [25]. In this study, our probeset was positioned at approximately FP1 and FP2, according to the international 10-20 system [26]. In this location, our probeset was placed on both the eyebrows, and LEDs and photodiodes were placed $1 \mathrm{~cm}$ above the forehead to remove the skin signal.

All participants performed olfactory-stimulated fNIRS in two different phases. The rest phase was performed for $40 \mathrm{~s}$, and the olfactory stimulation phase was performed for $20 \mathrm{~s}$. There were two types of olfactory stimulation with sniff stick pens (unscented and peppermint-scented; Burghart Screening 12 Test, MediSense, Netherlands) [27]. We conducted each test three times (total time of olfactory stimulation fNIRS: $3 \mathrm{~min}$ ). 


\section{Sample size calculation}

Since there has been no study on the direct relationship between olfactory-stimulated fNIRS and cognitive impairment, we calculated the sample size based on a previous similar study on the association between verbal fluency task-stimulated fNIRS and cognitive impairment [16]. Originally, we calculated that for two groups (CN versus $\mathrm{MCI}$ ) to have a $75 \%$ power to show a $40 \%$ oxygenation difference at a $5 \%$ significance level, we would need to enroll at least 15 participants in each group. Finally, considering the difficulty in recruiting patients with $\mathrm{AD}$, we included 55 participants with $\mathrm{CN}, 25$ with $\mathrm{MCI}$, and 16 with $\mathrm{AD}$.

\section{Statistical analysis}

Data was generated using Python, and in order to remove the noise generated by movement, the $\pm 1 \mathrm{mmol} \times \mathrm{mm} / \mathrm{l}$ channels that occurred simultaneously in oxygenated and deoxygenated hemoglobin were manually interpolated. For preprocessing of the concentration change of oxygenated and deoxygenated hemoglobin, high-frequency artifacts were excluded by applying a moving window of $3 \mathrm{~s}$ to the first raw data electrical signal. Then, to rule out a drift in fNIRS, we obtained a baseline value during the rest phase for $40 \mathrm{~s}$. In order to remove the system effect for each channel, the filter was processed below $0.4 \mathrm{~Hz}$ and then wavelet transform was performed. In addition, the data were scaled down by measuring the skin signal coming from a nearby channel and then removed from the original signal (C-NIRS algorithm). Then, the average change in values between the stimulation and rest phases for each participant was calculated and set as representative values.

Epidemiologic data are presented as mean and standard deviation (SD) or median and interquartile range. All statistical analyses were performed using SPSS (version 25.0; IBM Corp, Armonk, NY, USA) and R software version 3.1.1 (R Foundation, Vienna, Austria). An analysis of covariance was performed to assess between-group differences in oxygenation differences in the orbitofrontal cortex (CN versus $\mathrm{MCI}$ versus $\mathrm{AD}$ ), unadjusted in the crude model, adjusted for age and sex in model 1 , and adjusted for age, sex, education (continuous), household income (low, middle, and high), smoking (never or exsmoker and current smoker), and Charlson comorbidity index $(0,1$, and $\geq 2)$ in model $2[28,29]$. We performed linear regression models between stimulated oxygenation difference in the orbitofrontal cortex (continuous) and cognitive impairment-related outcomes (MMSE, SNSB, SUVR, and hippocampal volume; continuous) in model 2. Finally, we used the $\mathrm{C}$-statistic for the prediction model in the diagnosis of $\mathrm{AD}$ or $\mathrm{MCI}$ (dichotomized value), which was presented as the mean area under the receiver operator curve (AUC) value with 95\% confidence intervals (CIs). To test the reliability of our main findings, we analyzed the differential conditions using alternative MCI definitions (Jak/Bondi typical criteria), such as a $z$-score less than -1.5 on at least one of the SNSB tests and using another olfactory stimulation (leatherscented). Two-sided $P$-values $<.05$ were considered statistically significant.

\section{Results}

\section{Baseline characteristics}

A total of 103 elderly volunteers (age $>60$ years) with a suspected decline in cognitive function were screened, and 97 of them met the eligibility criteria. Candidates were excluded if they had any severe or life-threatening disease such as any malignancy or severe head trauma (excluded $n=0$ ), a physical nasal obstruction with an inability to smell (excluded $n=1$ ), or a serious mental problem that led to non-cooperation while answering the questionnaire (excluded $n=5$ ). For the overall trial, a total of 97 participants were recruited, of whom 55 (56.7\%) were $\mathrm{CN}$ (median age 74.0 years; female $50.9 \%$ ), 26 (26.7\%) were MCI patients (median age 74.0 years; female $50.0 \%$ ), and 16 (16.5\%) were AD patients (median age 76.5 years; female $43.8 \%$ ) (Table 1 ).

\section{Primary outcome}

Compared with $\mathrm{CN}$ participants (Table 2 and Fig. 1), olfactory-stimulated oxygenation in the orbitofrontal cortex was decreased in patients with MCI (model 2; adjusted mean difference, 10.81; 95\% CI, 5.27-16.36) and AD (model 2; adjusted mean difference 12.54; $95 \%$ CI 6.73-18.35). However, no differences were seen in the non-stimulated oxygenation levels in the orbitofrontal cortex in any of the groups.

\section{Secondary outcome}

Among the entire study population (Table 3 ), the olfactory-stimulated oxygenation difference in the orbitofrontal cortex was associated with MMSE scores $(a \beta$ 1.001; 95\% CI 0.540-1.463), SNSB language and related function scores $(a \beta 1.218 ; 95 \%$ CI $0.020-2.417)$, SNSB memory scores ( $a \beta 1.963 ; 95 \% C I 0.841-3.084)$, SNSB frontal/ executive function scores $(a \beta 1.715$; 95\% CI 0.401-3.029), amyloid PET SUVR $(a \beta-10.083$; 95\% $C I-19.063$ to -1.103 ), and MRI hippocampal volume ( $a \beta 0.002 ; 95 \%$ CI 0.001-0.004).

\section{Prediction model}

The AUC for the diagnosis of AD was 0.837 (95\% CI 0.753-0.921; sensitivity $100.0 \%$; specificity $61.7 \%$ ) using olfactory-stimulated oxygenation difference in the orbitofrontal cortex, 0.786 (95\% CI 0.656-0.917; sensitivity 
Table 1 Baseline characteristics of participants at enrollment $(n=97)$

\begin{tabular}{|c|c|c|c|}
\hline Variables & $\mathrm{CN}$ & $\mathrm{MCl}^{\mathrm{a}}$ & $A D$ \\
\hline Number (\%) & $55(56.7)$ & $26(26.8)$ & $16(16.5)$ \\
\hline Age, years, median (IQR) & $74.0(70.0-79.0)$ & $74.0(68.0-78.5)$ & $76.5(74.0-82.0)$ \\
\hline \multicolumn{4}{|l|}{ Body mass index, $\mathrm{kg} / \mathrm{m}^{2}, n(\%)$} \\
\hline$<25$ (normal) & 35 (63.6) & $18(69.2)$ & $12(75.0)$ \\
\hline$\geq 25$ (overweight or obese) & $20(36.4)$ & $8(30.8)$ & $4(12.5)$ \\
\hline Sex, female (\%) & $28(50.9)$ & $13(50.0)$ & $7(43.8)$ \\
\hline Education, years, median (IQR) & $9.0(6.0-14.0)$ & $12.0(6.0-12.25)$ & $11.0(8.0-14.8)$ \\
\hline \multicolumn{4}{|l|}{ Occupation, $n(\%)$} \\
\hline White collar/professional & $20(36.4)$ & $7(26.9)$ & $4(25.0)$ \\
\hline Blue collar & $27(49.1)$ & $16(61.5)$ & $9(56.3)$ \\
\hline Household/student/unemployed & $8(14.5)$ & $3(11.5)$ & $3(18.8)$ \\
\hline \multicolumn{4}{|l|}{ Household income, $n$ (\%) } \\
\hline Low (1-29 percentile) & $9(16.4)$ & $7(26.9)$ & $6(37.5)$ \\
\hline Middle (30-69 percentile) & $24(43.6)$ & $12(46.2)$ & $5(31.3)$ \\
\hline High (70-100 percentile) & $22(40.0)$ & $7(26.9)$ & $5(31.3)$ \\
\hline \multicolumn{4}{|l|}{ Smoking, $n(\%)$} \\
\hline Never or ex-smoker & $52(94.5)$ & $26(100.0)$ & $14(87.5)$ \\
\hline Current smoker & $3(5.5)$ & $0(0.0)$ & $2(12.5)$ \\
\hline \multicolumn{4}{|l|}{ Charlson comorbidity index, $n(\%)$} \\
\hline 0 & $20(36.4)$ & $9(34.6)$ & $6(37.5)$ \\
\hline 1 & $24(43.6)$ & $11(42.3)$ & $5(31.3)$ \\
\hline$\geq 2$ & $11(20.0)$ & $6(23.1)$ & $5(31.3)$ \\
\hline APOE4 carrier, $n(\%)$ & $14(21.2)$ & $18(85.7)$ & $11(68.8)$ \\
\hline Mini-Mental State Examination, median (range) & $28.0(24.0-30.0)$ & $26.0(22.0-30.0)$ & $20.0(10.0-24.0)$ \\
\hline \multicolumn{4}{|l|}{ Cognitive measures (composite $z$-score), mean (SD) } \\
\hline SNSB attention & $-0.07(0.92)$ & $-0.52(0.79)$ & $-0.61(0.94)$ \\
\hline SNSB language and related function & $0.51(0.65)$ & $0.02(1.22)$ & $-2.18(3.22)$ \\
\hline SNSB visuospatial function & $1.01(0.68)$ & $0.17(1.69)$ & $-2.45(5.00)$ \\
\hline SNSB memory & $0.73(1.08)$ & $-0.64(1.46)$ & $-2.73(1.30)$ \\
\hline SNSB frontal/executive function & $0.55(0.80)$ & $-0.47(1.06)$ & $-2.38(1.53)$ \\
\hline Amyloid PET (standard uptake value ratio), mean (SD) & $1.13(0.16)$ & $1.27(0.26)$ & $1.39(0.20)$ \\
\hline Hippocampal volume, $\mathrm{cm}^{3}$, mean (SD) & $7.68(0.99)$ & $7.01(1.40)$ & $6.04(1.27)$ \\
\hline
\end{tabular}

Abbreviations: $A D$ Alzheimer disease, $C N$ cognitively normal, IQR interquartile range, $M C l$ mild cognitive impairment, $S D$ standard deviation, $S N S B$ Seoul Neuropsychological Screening Battery

${ }^{\text {a }}$ The diagnostic criteria for $\mathrm{MCl}$ were based on the Jak/Bondi comprehensive criteria

86.7\%; specificity $81.3 \%$ ) using amyloid PET SUVR, and 0.810 (95\% CI 0.673-0.947; sensitivity $93.3 \%$; specificity 57.1\%) using MRI hippocampal volume (Table 4). The AUC for the diagnosis of both AD and MCI was 0.909 ( $95 \%$ CI $0.848-0.971$; sensitivity $84.7 \%$; specificity $94.4 \%$ ) using olfactory-stimulated oxygenation difference in the orbitofrontal cortex, 0.793 (95\% CI 0.694-0.893; sensitivity $89.8 \%$; specificity $63.9 \%$ ) using amyloid PET SUVR, and 0.758 (95\% CI 0.644-0.871; sensitivity 98.1\%; specificity $66.7 \%$ ) using MRI hippocampal volume. Finally, the AUC for $\mathrm{MCI}$ diagnosis excluding patients with $\mathrm{AD}$ was the highest using olfactory-stimulated oxygenation difference in the orbitofrontal cortex (total $n=81 ; 0.903$; 95\% CI 0.836-0.970; sensitivity $95.2 \%$; specificity $85.0 \%$ ), compared with the amyloid PET SUVR $(0.760 ; 95 \%$ CI $0.629-0.891$; sensitivity $90.0 \%$; specificity $56.1 \%)$ and MRI hippocampal volume (0.718; 95\% CI 0.570-0.867; sensitivity $52.4 \%$; specificity $91.5 \%$ ) (Fig. 2). Finally, similar patterns of association were observed in the sensitivity analyses of differential conditions using alternative MCI definitions (Jak/Bondi typical criteria; Table S1 and S2) and another olfactory stimulation (leather-scented; Table S3). 
Table 2 Association between stimulated oxygenation difference in the orbitofrontal cortex and cognitive impairment (primary endpoint)

\begin{tabular}{|c|c|c|c|c|c|}
\hline $\begin{array}{l}\text { Oxygenation difference in } \\
\text { the orbitofrontal cortex }\end{array}$ & Model & $\mathrm{CN}$ & $\mathrm{MCl}^{\mathrm{c}}$ & $A D$ & $P$ trend \\
\hline \multirow[t]{3}{*}{ Olfactory stimulation } & Mean $(95 \%$ Cl) & 5.94 (3.13 to 8.74$)$ & $-0.22(-2.48$ to 2.04$)$ & $-3.96(-5.69$ to -2.23$)$ & \\
\hline & Adjusted mean difference (model $1^{\mathrm{a}}$ ) & 1.00 (reference) & $6.12(2.12$ to 10.13$)$ & $9.93(5.10$ to 14.76$)$ & $<0.001$ \\
\hline & Adjusted mean difference (model $2^{b}$ ) & 1.00 (reference) & $6.83(3.15$ to 10.51$)$ & $9.92(5.72$ to 14.13$)$ & $<0.001$ \\
\hline \multirow[t]{3}{*}{ None } & Mean $(95 \% C l)$ & $1.63(-1.67$ to 4.93$)$ & $1.92(-2.46$ to 6.30$)$ & $0.93(-1.71$ to 3.57$)$ & \\
\hline & Adjusted mean difference (model $1^{\mathrm{a}}$ ) & 1.00 (reference) & $-0.47(-5.57$ to 4.63$)$ & $1.32(-4.83$ to 7.46$)$ & 0.872 \\
\hline & Adjusted mean difference (model $2^{b}$ ) & 1.00 (reference) & $-0.39(-7.05$ to 6.26$)$ & $0.51(-7.11$ to 8.12$)$ & 0.870 \\
\hline
\end{tabular}

Numbers in bold indicate statistically significant associations $(P<0.05)$

Abbreviations: $A D$ Alzheimer's disease, $C N$ cognitively normal, $M C l$ mild cognitive impairment

${ }^{a}$ Model 1 was adjusted for age and sex

${ }^{\mathrm{b}}$ Model 2 was adjusted for age, sex, education (continuous), household income (low, middle, and high), smoking (never or ex-smoker and current smoker), and Charlson comorbidity index $(0,1$, and $\geq 2)$

' The diagnostic criteria for $\mathrm{MCl}$ were based on the Jak/Bondi comprehensive criteria

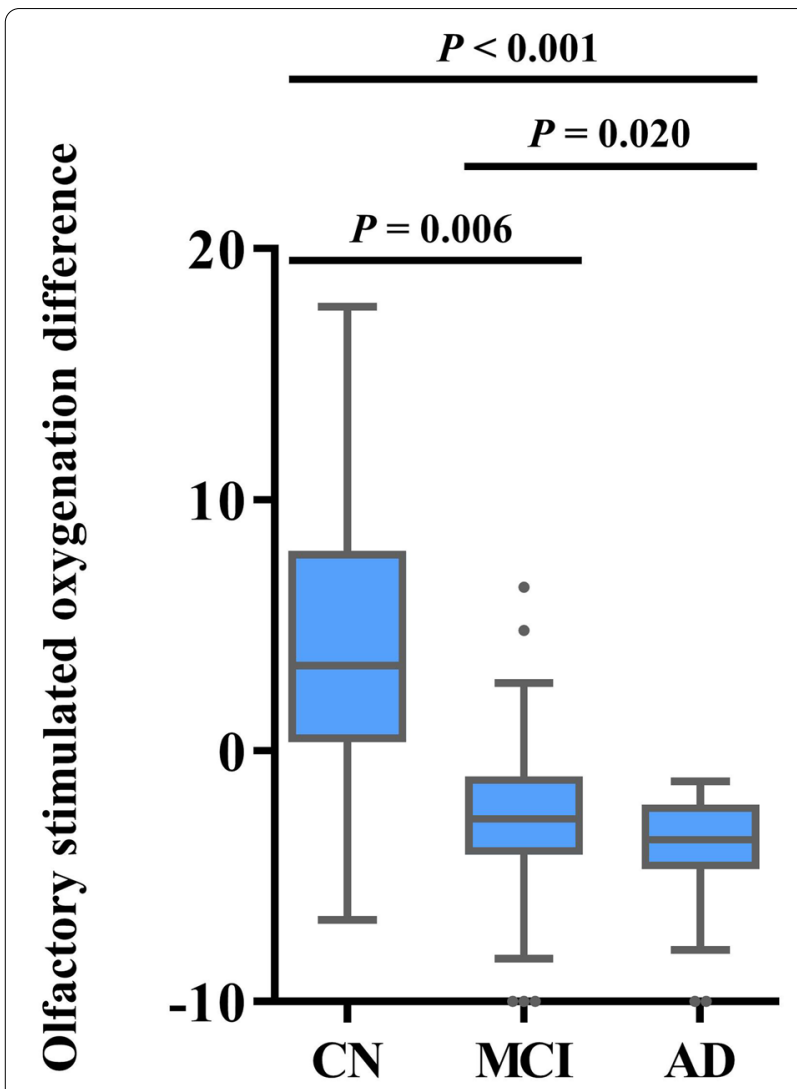

Fig. 1 Olfactory-stimulated oxygenation in the orbitofrontal cortex in patients with $\mathrm{CN}, \mathrm{MCl}$, and $\mathrm{AD}$. The top and bottom of each box indicate the interquartile range; the I bars represent 1.5 times the interquartile range; the horizontal line inside each box represents the median; the circles represent outliers. These values were analyzed using the Wilcoxon rank sum test

\section{Discussion}

\section{Findings of our study}

Through a patient-level, single-group diagnostic trial, this is the first well-designed study for early detection of MCI and/or AD using novel fNIRS diagnostic techniques. We found that olfactory-stimulated oxygenation difference in the orbitofrontal cortex measured by fNIRS was associated with cognitive impairment, and its association was more pronounced with cognitive severity, whereas non-stimulated oxygenation difference was not associated with any cognitive impairment. In addition, the olfactory-stimulated oxygenation difference was associated with MMSE scores, SNSB language and related function scores, SNSB memory scores, SNSB frontal/executive function scores, SUVR from amyloid PET, and hippocampal volume from MRI. Finally, our results indicated that olfactory-stimulated oxygenation difference in the orbitofrontal cortex had diagnostic superiority for $\mathrm{MCI}$ and/or AD compared with amyloid PET or MRI scan.

\section{Comparison with previous studies}

Previous diagnostic studies of MCI have used the fNIRS approach. In 2006, a preliminary study using the fNIRS approach during the verbal fluency task suggested the first potential diagnostic evidence for $\mathrm{AD}$ (total $n=47$ ) [12]. Many researchers have provided several methods of stimulation using the fNIRS approach, including dualtask walking $(n=16)$ [13], resting state $(n=21)$ [14], N-back task $(n=24)$ [15], verbal fluency task $(n=61)$ [16], and memory and visuospatial test $(n=11)$ [17]. However, these previous studies have been conducted with small sample sizes and did not include comparisons with other diagnostic tests such as MRI, PET, or APOE genotypes, which may lead to difficulties in generating 
Table 3 Association between stimulated oxygenation difference in the orbitofrontal cortex and cognitive impairment-related outcomes (secondary endpoints)

\begin{tabular}{|c|c|c|c|c|c|}
\hline \multirow[t]{2}{*}{ Variables } & \multirow{2}{*}{$\begin{array}{l}\text { Oxygenation difference } \\
\text { in the orbitofrontal } \\
\text { cortex }\end{array}$} & \multicolumn{2}{|l|}{ Crude model } & \multicolumn{2}{|l|}{ Adjusted model $^{\mathrm{a}}$} \\
\hline & & $\beta(95 \% C l)$ & $P$ value & $\beta(95 \% C l)$ & $P$ value \\
\hline \multirow[t]{2}{*}{ Mini-Mental State Examination } & Olfactory stimulation & 0.957 ( 0.512 to 1.403$)$ & $<0.001$ & 1.001 (0.540 to 1.463 ) & $<0.001$ \\
\hline & None & $-0.110(-0.680$ to 0.461$)$ & 0.704 & $0.007(-0.569$ to 0.583$)$ & 0.981 \\
\hline \multirow[t]{2}{*}{ SNSB attention } & Olfactory stimulation & $1.654(-0.426$ to 3.734$)$ & 0.118 & $1.828(-0.315$ to 3.970$)$ & 0.179 \\
\hline & None & $-0.618(-3.098$ to 1.861$)$ & 0.622 & $-0.440(-2.916$ to 2.037$)$ & 0.725 \\
\hline \multirow[t]{2}{*}{ SNSB language and related function } & Olfactory stimulation & 1.292 (0.129 to 2.454$)$ & 0.030 & $1.218(0.020$ to 2.417$)$ & 0.046 \\
\hline & None & $-0.795(-2.192$ to 0.601$)$ & 0.261 & -0.961 ( -2.367 to 0.445$)$ & 0.178 \\
\hline \multirow[t]{2}{*}{ SNSB visuospatial function } & Olfactory stimulation & $0.679(-0.076$ to 1.434$)$ & 0.077 & $0.626-0.155$ to 1.408$)$ & 0.115 \\
\hline & None & $-0.105(-1.006$ to 0.796$)$ & 0.818 & $-0.072(-0.973$ to 0.829$)$ & 0.875 \\
\hline \multirow[t]{2}{*}{ SNSB memory } & Olfactory stimulation & $1.830(0.802$ to 2.858$)$ & 0.001 & $1.963(0.841$ to 3.084$)$ & 0.001 \\
\hline & None & -0.394 ( -1.676 to 0.888$)$ & 0.544 & $-0.361-1.717$ to 0.994$)$ & 0.598 \\
\hline \multirow[t]{2}{*}{ SNSB frontal/executive function } & Olfactory stimulation & 1.817 (0.557 to 3.078$)$ & 0.005 & 1.715 (0.401 to 3.029$)$ & 0.011 \\
\hline & None & $-0.323(-1.865$ to 1.219$)$ & 0.678 & -0.361 ( -1.908 to 1.186$)$ & 0.644 \\
\hline \multirow[t]{2}{*}{ Standard uptake value ratio } & Olfactory stimulation & $-9.462(-18.062$ to -0.862$)$ & 0.031 & $-10.083(-19.063$ to -1.103$)$ & 0.028 \\
\hline & None & 0.391 (-9.878 to 10.660) & 0.076 & $-2.514(-12.817$ to 7.790$)$ & 0.629 \\
\hline \multirow[t]{2}{*}{ Hippocampal volume } & Olfactory stimulation & $0.002(0.001$ to 0.003$)$ & 0.007 & $0.002(0.001$ to 0.004$)$ & 0.005 \\
\hline & None & -0.001 ( -0.003 to 0.001$)$ & 0.278 & -0.001 ( -0.002 to 0.001$)$ & 0.543 \\
\hline
\end{tabular}

Numbers in bold indicate statistically significant associations $(P<0.05)$

Abbreviations: AD Alzheimer's disease, CN cognitively normal, MCl mild cognitive impairment, SNSB Seoul Neuropsychological Screening Battery

${ }^{a}$ Risk factors were adjusted for age, sex, education (continuous), household income (low, middle, and high), smoking (never or ex-smoker and current smoker), and Charlson comorbidity index $(0,1$, and $\geq 2)$

robust results and the interpretation of results. In addition, most of the stimulation methods used increased the burden of medical providers and subjects, unlike the olfactory stimulation method. Furthermore, our report is the first to reveal the diagnostic superiority of olfactorystimulated fNIRS through direct comparison with other conventional imaging tests.
Furthermore, previous studies suggest that response patterns of olfactory stimulation are reflected in various modalities, such as fMRI [30] and EEG [31]. Changes in EEG patterns by olfactory stimulation are particularly characteristic in the FP1 and FP2 areas [31], which is similar to our results using the fNIRS approach in these areas.

Table 4 C-statistic for the prediction model in the diagnosis of AD or MCl ${ }^{b}$

\begin{tabular}{|c|c|c|c|}
\hline & AUC & Sensitivity (\%) & Specificity (\%) \\
\hline \multicolumn{4}{|l|}{ Prediction model as AD } \\
\hline Olfactory-stimulated oxygenation difference in the orbitofrontal cortex & 0.819 (0.736 to 0.902$)$ & 93.8 & 67.9 \\
\hline Standard uptake value ratio (amyloid PET) & 0.786 (0.656 to 0.917$)$ & 93.3 & 57.1 \\
\hline Hippocampal volume (MRI) & 0.810 (0.673 to 0.947$)$ & 86.7 & 81.3 \\
\hline \multicolumn{4}{|l|}{ Prediction model as $A D$ and $M C l$} \\
\hline Olfactory-stimulated oxygenation difference in the orbitofrontal cortex & 0.873 (0.800 to 0.945$)$ & 88.1 & 81.8 \\
\hline Standard uptake value ratio (amyloid PET) & 0.745 (0.640 to 0.850$)$ & 80.0 & 59.6 \\
\hline Hippocampal volume & 0.733 (0.621 to 0.846$)$ & 61.0 & 92.6 \\
\hline \multicolumn{4}{|l|}{ Prediction model as MCI (excluded patients with AD) ${ }^{\mathrm{a}}$} \\
\hline Olfactory-stimulated oxygenation difference in the orbitofrontal cortex & 0.852 (0.764 to 0.939$)$ & 84.6 & 81.8 \\
\hline Standard uptake value ratio (amyloid PET) & 0.690 (0.557 to 0.823$)$ & 52.0 & 80.8 \\
\hline Hippocampal volume (MRI) & 0.659 (0.515 to 0.804$)$ & 50.0 & 88.9 \\
\hline
\end{tabular}

Abbreviations: $A D$ Alzheimer's disease, $A \cup C$ area under the curve, $C N$ cognitively normal, $M C l$ mild cognitive impairment

${ }^{a}$ We excluded 16 patients with $A D$; therefore, the sample number for this analysis is 81

${ }^{\mathrm{b}}$ The diagnostic criteria for $\mathrm{MCl}$ were based on the Jak/Bondi comprehensive criteria 


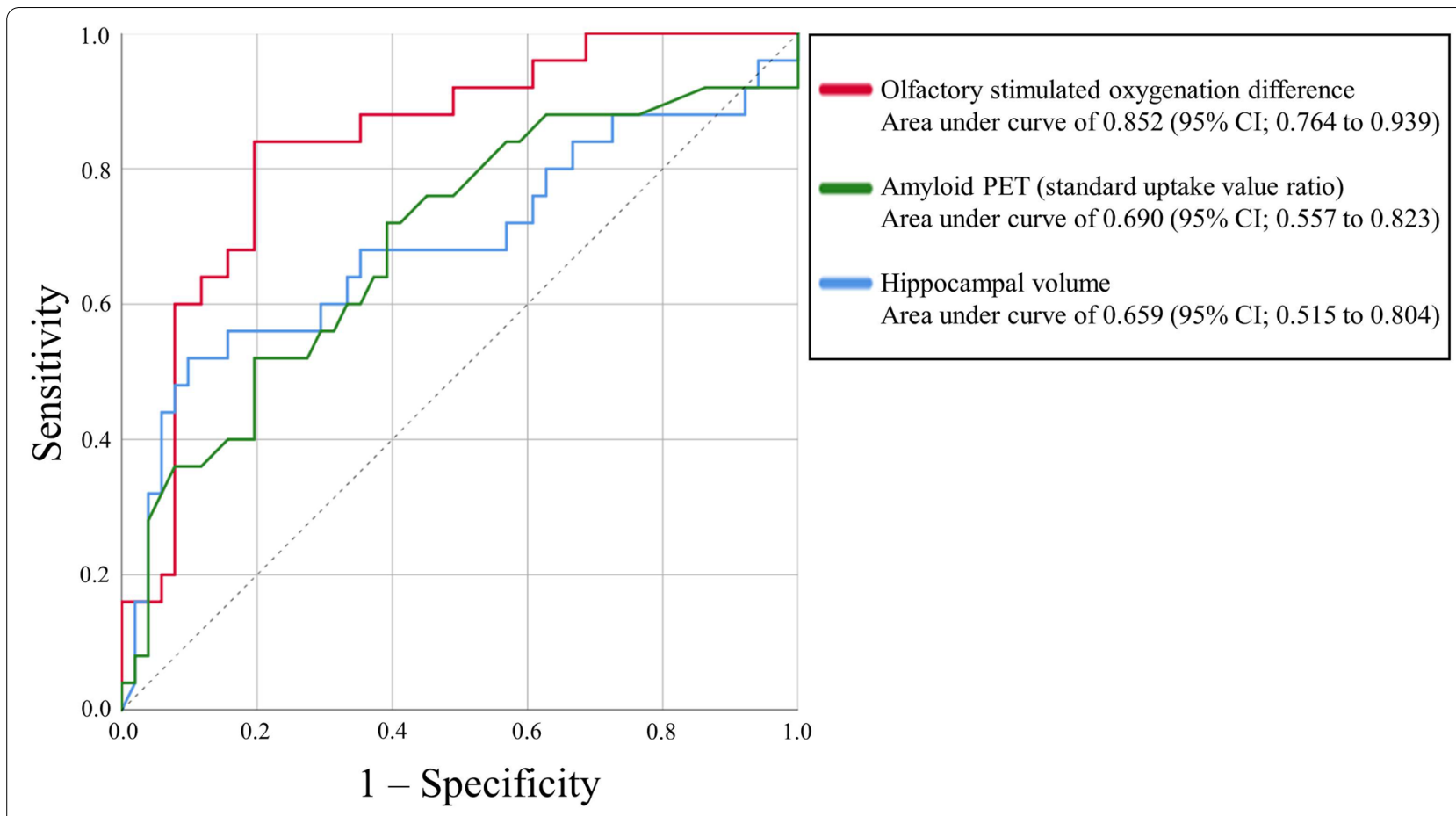

Fig. 2 Receiver operating characteristic curves for various models of diagnosis of mild cognitive impairment in our cohort excluded patients with Alzheimer disease $(n=81)$

\section{Possible explanations of our results}

Previous studies have reported that olfactory dysfunction is an early symptom of cognitive impairment [32]. Previous post-mortem studies suggest that pathological changes in patients with AD involved pathological changes in the anterior olfactory nucleus and the olfactory bulb during the early stage of the disease, even before clinical symptoms manifest [33]. The Braak and Tredici hypothesis indicated that olfactory function might be vulnerable to $A D$ and may affect disease progression [34]. Moreover, early neurodegenerative change patterns have been well established in $\mathrm{AD}$ according to their relationship with the olfactory bulb, prepiriform cortex, amygdala, entorhinal cortex, basal forebrain, raphe nuclei, locus coeruleus, and hippocampus [7]. Furthermore, previous epidemiologic studies suggest that olfactory function could predict neurodegeneration and decline in cognitive function through MRI [35] and PET [36] image findings and cognitive assessment questionnaires [37]. Therefore, many researchers have suggested that the olfactory function is a potential biomarker [7]. Furthermore, several epidemiologic studies have suggested that odor identification can predict the development of $\mathrm{MCI}$ [38]. Our study provides objective indicators for novel diagnostic tools along with the biological and epidemiological backgrounds linking olfactory function and
AD. However, our study was preliminary with a small sample size and participants of only Asian ethnicity, and therefore, there are limitations of generalization and reproducibility.

\section{Policy implication}

Our novel method can provide a non-invasive, high portability tool with lower cost, low radiation exposure, short examination time, fewer constraints on elderly patients who have difficulty sitting down for a long time or filling out questionnaires for examination, and excellent diagnostic superiority. A previous study suggested that the fNIRS approach can offer cognitive function assessment in resource-poor, rural communities [39], and our findings may reflect the value of this diagnostic tool that can be easily used not only in developed countries but also in underdeveloped countries.

\section{Strengths and limitations}

Our study had some limitations. First, due to channel number restrictions to increase portability, the area of the fNIRS measurement was only the frontal cortex. Although our method provided excellent diagnostic results, olfactory-stimulated whole-head fNIRS measurement might be used to confirm the potential relationship and brain functional connectivity between other brain regions and $\mathrm{AD}$ [40]. Second, there are well-established 
ethnic differences in dementia risk [41] and our results are aimed at only Asians (Koreans); therefore, international research on ethnic differences in $\mathrm{AD}$ is needed for diagnostic validation. Third, although our study was an intervention trial, and for fNIRS to be used as a biomarker, we need a longitudinal study that includes repeated tests. Despite these limitations, our study has some strengths. Unlike previous studies, we enhanced the reproducibility and generalizability of our results through various imaging studies, $A P O E$ genotyping, and a larger sample size. In addition, we have provided diagnostic insights related to AD because we have conducted interventional trials from a medical perspective rather than from an engineering perspective. Finally, although we calculated the appropriate sample size for a diagnostic trial, we should be careful with the interpretation of the main results, which could be overestimated and skewed due to a small sample size. Therefore, future large-scale randomization diagnostic trials such as international multicenter trials are warranted.

\section{Conclusions}

Through a patient-level, single-group, diagnostic intervention trial, this is the first well-designed study for the early detection of $\mathrm{MCI}$ and/or $\mathrm{AD}$ using novel olfactory-stimulated fNIRS diagnostic techniques. We found that olfactory-stimulated oxygenation difference in the orbitofrontal cortex had diagnostic superiority for $\mathrm{MCI}$ and/or AD, compared with amyloid PET or MRI scan. Our well-designed diagnostic trial suggests that the novel olfactory-stimulated fNIRS diagnostic technique may be a potential diagnostic tool for patients with MCI and/or AD and that large-scale randomized longitudinal trials of the diagnostic use of olfactory-stimulated ANIRS for AD are warranted.

\section{Supplementary Information}

The online version contains supplementary material available at https://doi. org/10.1186/s13195-022-00978-w.

Additional file 1: Supplementary Methods and Results.

\section{Acknowledgements}

Not applicable.

\section{Authors' contributions}

$J K, D K Y, K H L$, and JWK contributed to the conception and design of the study. KYC, JJL, and NK contributed to the acquisition and analysis of the data. DKY contributed to drafting the text or preparing the figures. All authors critically revised the manuscript. The authors read and approved the final manuscript, and DKY, JGK and KHL proofread and approved the final manuscript.

\section{Funding}

This work was supported by a grant of the MD-PhD/Medical Scientist Training Program through the Korea Health Industry Development Institute (KHIDI) funded by the Ministry of Health \& Welfare and the Ministry of Health \& Brain Research Program funded by the National Research Foundation of Korea [NRF2016M3C7A1905475]; KBRI basic research program through Korea Brain Research
Institute funded by Ministry of Science and ICT [21-BR-03-05]; the Original Technology Research Program for Brain Science of the National Research Foundation (NRF-2014M3C7A1046041); and the Institute of Information \& communications Technology Planning \& Evaluation (IITP) grant (MSIT; No.2020-0-01969), funded by the Korean government, MSIT. The funders had no role in the study design, data collection, data analysis, data interpretation, or writing of the report.

\section{Availability of data and materials}

The datasets used and analyzed during the current study are available from the corresponding author on reasonable request.

\section{Declarations}

\section{Ethics approval and consent to participate}

The study protocol was approved by the Institutional Review Board of the Gwangju Institute of Science and Technology (20210115-HR-58-01-02). The trial was registered with the Clinical Research Information Service of the Republic of Korea (CRIS number: KCT0006197). Our study adhered to the tenets of the Declaration of Helsinki.

\section{Consent for publication}

Not applicable.

\section{Competing interests}

The authors declare that they have no competing interests.

\section{Author details}

${ }^{1}$ Department of Biomedical Science and Engineering, Gwangju Institute of Science and Technology, Gwangju, Republic of Korea. ${ }^{2}$ Medical Science Research Institute, Kyung Hee University College of Medicine, Seoul, Republic of Korea. ${ }^{3}$ Department of Data Science, Sejong University College of Software Convergence, Seoul, Republic of Korea. ${ }^{4}$ Gwangju Alzheimer's \& Related Dementia Cohort Research Center, Chosun University, Gwangju, Republic of Korea. ${ }^{5}$ Kolab Inc., Gwangju, Republic of Korea. ${ }^{6}$ Department of Neuropsychiatry, Seoul National University Hospital, Seoul, Republic of Korea. ${ }^{7}$ Gwangju Alzheimer's \& Related Dementia Cohort Research Center, Department of Biomedical Science, Chosun University, Gwangju 61452, Republic of Korea. ${ }^{8}$ Korea Brain Research Institute, Daegu, Republic of Korea.

Received: 1 October 2021 Accepted: 8 February 2022

Published online: 08 March 2022

\section{References}

1. Rajan K, Weuve J, Barnes L, McAninch E, Wilson R, Evans D. Population estimate of people with clinical Alzheimer's disease and mild cognitive impairment in the United States (2020-2060). Alzheimers Dement. 2021;17(12):1966-75.

2. Seo EH, Lim HJ, Yoon HJ, Choi KY, Lee JJ, Park JY, et al. Visuospatial memory impairment as a potential neurocognitive marker to predict tau pathology in Alzheimer's continuum. Alzheimers Res Ther. 2021;13(1):167.

3. Attems J, Walker $L$, Jellinger KA. Olfactory bulb involvement in neurodegenerative diseases. Acta Neuropathol. 2014;127(4):459-75.

4. Wilson RS, Arnold SE, Schneider JA, Tang Y, Bennett DA. The relationship between cerebral Alzheimer's disease pathology and odour identification in old age. J Neurol Neurosurg Psychiatry. 2007;78(1):30-5.

5. Alotaibi M, Lessard-Beaudoin M, Loudghi A, Chouinard-Watkins R, Plourde M, Calon F, et al. Decrease in olfactory cortex volume and alterations in caspase expression in the olfactory bulb in the pathogenesis of Alzheimer's disease. Alzheimers Dement. 2020;16(S6):e039789.

6. Attems J, Lintner F, Jellinger KA. Olfactory involvement in aging and Alzheimer's disease: an autopsy study. J Alzheimer's Dis. 2005;7(2):149-57 discussion 173-180.

7. Murphy C. Olfactory and other sensory impairments in Alzheimer disease. Nat Rev Neurol. 2019;15(1):11-24.

8. Yoo SJ, Lee JH, Kim SY, Son G, Kim JY, Cho B, et al. Differential spatial expression of peripheral olfactory neuron-derived BACE1 induces olfactory impairment by region-specific accumulation of $\beta$-amyloid oligomer. Cell Death Dis. 2017;8(8):e2977. 
9. Ehlis AC, Schneider S, Dresler T, Fallgatter AJ. Application of functional near-infrared spectroscopy in psychiatry. Neurolmage. 2014;85(Pt 1):478-88.

10. Ho RC, Sharma VK, Tan BY, Ng AY, Lui Y-S, Husain SF, et al. Comparison of brain activation patterns during olfactory stimuli between recovered COVID-19 patients and healthy controls: a functional near-infrared spectroscopy (fNIRS) study. Brain Sci. 2021;11(8):968.

11. Li R, Rui G, Chen W, Li S, Schulz PE, Zhang Y. Early detection of Alzheimer's disease using non-invasive near-infrared spectroscopy. Front Aging Neurosci. 2018;10:366

12. Arai H, Takano M, Miyakawa K, Ota T, Takahashi T, Asaka H, et al. A quantitative near-infrared spectroscopy study: a decrease in cerebral hemoglobin oxygenation in Alzheimer's disease and mild cognitive impairment. Brain Cogn. 2006;61(2):189-94.

13. Doi T, Makizako H, Shimada H, Park H, Tsutsumimoto K, Uemura K, et al. Brain activation during dual-task walking and executive function among older adults with mild cognitive impairment: a fNIRS study. Aging Clin Exp Res. 2013;25(5):539-44.

14. Viola S, Viola P, Buongarzone MP, Fiorelli L, Litterio P. Tissue oxygen saturation and pulsatility index as markers for amnestic mild cognitive impairment: NIRS and TCD study. Clin Neurophysiol. 2013;124(5):851-6.

15. Niu HJ, Li X, Chen YJ, Ma C, Zhang JY, Zhang ZJ. Reduced frontal activation during a working memory task in mild cognitive impairment: a non-invasive near-infrared spectroscopy study. CNS Neurosci Therapeut. 2013;19(2):125-31.

16. Yap KH, Ung WC, Ebenezer EGM, Nordin N, Chin PS, Sugathan S, et al. Visualizing hyperactivation in neurodegeneration based on prefrontal oxygenation: a comparative study of mild Alzheimer's disease, mild cognitive impairment, and healthy controls. Front Aging Neurosci. 2017;9:287.

17. Perpetuini D, Chiarelli AM, Cardone D, Filippini C, Bucco R, Zito M, et al. Complexity of frontal cortex fNIRS can support Alzheimer disease diagnosis in memory and visuo-spatial tests. Entropy (Basel). 2019;21(1):26.

18. Ahn H-J, Chin J, Park A, Lee BH, Suh MK, Seo SW, et al. Seoul neuropsychological screening battery-dementia version (SNSB-D): a useful tool for assessing and monitoring cognitive impairments in dementia patients. J Korean Med Sci. 2010;25(7):1071-6.

19. Chin J, Park J, Yang S-J, Yeom J, Ahn Y, Baek MJ, et al. Re-standardization of the Korean-instrumental activities of daily living (K-IADL): clinical usefulness for various neurodegenerative diseases. Dement Neurocogn Disord. 2018;17(1):11-22.

20. Jak AJ, Bondi MW, Delano-Wood L, Wierenga C, Corey-Bloom J, Salmon DP, et al. Quantification of five neuropsychological approaches to defining mild cognitive impairment. Am J Geriatr Psych. 2009;17(5):368-75.

21. Maass A, Landau S, Baker SL, Horng A, Lockhart SN, La Joie R, et al. Comparison of multiple tau-PET measures as biomarkers in aging and Alzheimer's disease. Neurolmage. 2017;157:448-63.

22. Clark CM, Schneider JA, Bedell BJ, Beach TG, Bilker WB, Mintun MA, et al. Use of florbetapir-PET for imaging beta-amyloid pathology. Jama. 2011;305(3):275-83.

23. Vemuri P, Lowe VJ, Knopman DS, Senjem ML, Kemp BJ, Schwarz CG, et al. Tau-PET uptake: regional variation in average SUVR and impact of amyloid deposition. Alzheimers Dement (Amst). 2016;6:21-30.

24. McKhann GM, Knopman DS, Chertkow H, Hyman BT, Jack CR Jr, Kawas $\mathrm{CH}$, et al. The diagnosis of dementia due to Alzheimer's disease: recommendations from the National Institute on Aging-Alzheimer's association workgroups on diagnostic guidelines for Alzheimer's disease. Alzheimers Dement. 2011;7(3):263-9.

25. Metzger FG, Schopp B, Haeussinger FB, Dehnen K, Synofzik M, Fallgatter AJ, et al. Brain activation in frontotemporal and Alzheimer's dementia: a functional near-infrared spectroscopy study. Alzheimers Res Ther. 2016:8(1):56.

26. Jasper $\mathrm{H}$. Report of the committee on methods of clinical examination in electroencephalography. Electroencephalogr Clin Neurophysiol. 1958;10:370-5.

27. Yon DK, Lee SW, Ha EK, Lee KS, Jung YH, Jee HM, et al. Serum lipid levels are associated with allergic rhinitis, nasal symptoms, peripheral olfactory function, and nasal airway patency in children. Allergy. 2018;73(9):1905-8.

28. Lee SW, Kim SY, Moon SY, Yang JM, Ha EK, Jee HM, et al. Estimating COVID-19 infection and severity risks in patients with chronic rhinosinusitis: a Korean nationwide cohort study. J Allergy Clin Immunol Pract. 2021;9(6):2262-2271.e2262.

29. Ha J, Lee SW, Yon DK. Ten-year trends and prevalence of asthma, allergic rhinitis, and atopic dermatitis among the Korean population, 2008-2017. Clin Exper Pediatr. 2020;63(7):278-83.

30. Zhang $H$, Ji D, Yin J, Wang Z, Zhou Y, Ni H, et al. Olfactory fMRI activation pattern across different concentrations changes in Alzheimer's disease. Front Neurosci. 2019;13:786.

31. Iravani B, Arshamian A, Ohla K, Wilson DA, Lundström JN. Non-invasive recording from the human olfactory bulb. Nat Commun. 2020;11(1):648.

32. Zou Y-M, Lu D, Liu L-P, Zhang H-H, Zhou Y-Y. Olfactory dysfunction in Alzheimer's disease. Neuropsychiatr Dis Treat. 2016;12:869-75.

33. Ohm TG, Braak H. Olfactory bulb changes in Alzheimer's disease. Acta Neuropathol. 1987;73(4):365-9.

34. Braak H, Del Tredici K. The pathological process underlying Alzheimer's disease in individuals under thirty. Acta Neuropathol. 2011;121(2):171-81.

35. Dintica CS, Marseglia A, Rizzuto D, Wang R, Seubert J, Arfanakis K, et al. Impaired olfaction is associated with cognitive decline and neurodegeneration in the brain. Neurology. 2019;92(7):e700-9.

36. Yoo HS, Chung SJ, Lee YH, Ye BS, Sohn YH, Lee PH. Association between olfactory deficit and motor and cognitive function in Parkinson's disease. JMD. 2020;13(2):133-41.

37. Yahiaoui-Doktor M, Luck T, Riedel-Heller SG, Loeffler M, Wirkner K, Engel C. Olfactory function is associated with cognitive performance: results from the population-based LIFE-adult-study. Alzheimers Res Ther. 2019;11(1):43.

38. Woodward MR, Amrutkar CV, Shah HC, Benedict RH, Rajakrishnan S, Doody RS, et al. Validation of olfactory deficit as a biomarker of Alzheimer disease. Neurol Clin Pract. 2017;7(1):5-14.

39. Lloyd-Fox S, Papademetriou M, Darboe MK, Everdell NL, Wegmuller R, Prentice AM, et al. Functional near infrared spectroscopy (fNIRS) to assess cognitive function in infants in rural Africa. Sci Rep. 2014;4:4740.

40. Nguyen T, Kim M, Gwak J, Lee JJ, Choi KY, Lee KH, et al. Investigation of brain functional connectivity in patients with mild cognitive impairment: a functional near-infrared spectroscopy (fNIRS) study. J Biophotonics. 2019;12(9):e201800298.

41. Shiekh SI, Cadogan SL, Lin LY, Mathur R, Smeeth L, Warren-Gash C. Ethnic differences in dementia risk: a systematic review and meta-analysis. J Alzheimer's Dis. 2021;80(1):337-55.

\section{Publisher's Note}

Springer Nature remains neutral with regard to jurisdictional claims in published maps and institutional affiliations.

Ready to submit your research? Choose BMC and benefit from

- fast, convenient online submission

- thorough peer review by experienced researchers in your field

- rapid publication on acceptance

- support for research data, including large and complex data types

- gold Open Access which fosters wider collaboration and increased citations

- maximum visibility for your research: over $100 \mathrm{M}$ website views per year

At BMC, research is always in progress.

Learn more biomedcentral.com/submissions 